two protons to a $\mathrm{Be}^{--}$ion to form $\mathrm{BeH}_{2}$; the nuclear repulsion term is dominant and a linear molecule is formed (similarly $\mathrm{BH}_{3}$ will be trigonal and $\mathrm{CH}_{4}$ tetrahedral because these arrangements minimize the nuclear repulsion energy for any given internuclear separation). However, $\mathrm{O}^{--}$has ten electrons confined to a rather smaller volume than the six electrons in $\mathrm{Be}^{--}$( $\mathrm{O}$ is smaller than $\mathrm{Be}$ and we have assumed that this is also true for the corresponding negative ions). Thus the two protons encounter a much greater electron density in the region of the equilibrium internuclear separation causing the net attraction term to dominate, thereby reducing the $\mathrm{H}-\mathrm{O}-\mathrm{H}$ angle from $180^{\circ}$ to $104^{\circ}$ where the attractive force tending to superimpose the two nuclei balances the nuclear repulsion.

Thus for case (1) molecules ( $M$ belonging to the left-hand side of the periodic table) we have a common explanation of the bond angles which occur, but for case (2) molecules (i.e., $M$ on the right-hand side of the periodic table), we have to consider each one separately. The reason for the phenomenon of directed valence is conceptually much simpler than hitherto realized, but the hope of being able to calculate explicitly any case (2) bond angles becomes far more remote. One could formalize these arguments in terms of a suitable charge density function situated on the $M^{--}$ion and $1 s$ functions on the protons by ignoring the electronic kinetic energy. However, the kinetic energy does not remain constant during the molecule-forming process envisaged and such formalization is not therefore of much value. However, some generalizations, supported by experimental observation, about case (2) can be made: the greater the electron density around the central atom, the smaller will be the bond angle (compare the successive reduction in bond angle along the series $\mathrm{NH}_{3}, \mathrm{PH}_{3}, \mathrm{AsH}_{3}, \mathrm{SbH}_{3}$ ); also an atom in a low valence state may be case (2), but in a higher valence state may pass over into case (1) because of the increasing number of nuclear repulsions (e.g., $\mathrm{H}_{2} \mathrm{~S}$ is angular but in $\mathrm{SF}_{6}$ the fluorine atoms take up a symmetrical arrangement of minimum nuclear repulsion). Also, $\mathrm{SeCl}_{4}$ is case (1) whereas lower down the table $\mathrm{TeCl}_{4}$ is a case (2) molecule in accordance with the previous generalization (compare also the series $\mathrm{SF}_{6}-\mathrm{UF}_{6}$ ).

1 G. S. Gordadse, Z. Physik 99, 287 (1936).

2 D. R. Bates and T. R. Carson, Proc. Roy. Soc. (London) A234, 207 (1956).

\section{Polarization of Anthracene Crystal Fluorescence}

J. FERguson AND W. G. SCHNeIder Division of Pure Chemistry, National Research Council, Ottawa, Canada

(Received August 6, 1956)

$\mathrm{T}$ HE absorption spectrum of crystalline anthracene has aroused considerable attention recently in view of the current theory of molecular crystal spectra. ${ }^{1}$ The $3800 \mathrm{~A}$ system has been investigated by Craig and Hobbins ${ }^{2}$ and the reason for the departure from the "oriented gas" polarization ratio interpreted by Craig ${ }^{3}$ as due to crystal induced mixing with the higher ' $B_{3}$, molecular state. More recently Bree and Lyons ${ }^{4}$ have examined the polarization properties of this system and their results have substantiated the theory developed by Craig.

Although the absorption spectrum has been thoroughly investigated the fluorescence spectrum has received little attention. Obreimov, Prikhotjko, and Shabaldas, ${ }^{5}$ Pesteil and Barbaron, ${ }^{6}$ and Sidman ${ }^{7}$ have photographed the spectrum from single crystals at temperatures varying from $4^{\circ} \mathrm{K}$ to $20^{\circ} \mathrm{K}$. However, no attempt has been made to measure the polarization properties of this fluorescence with the exception of the work of Ganguly and Choudhuri ${ }^{8}$ which will be mentioned later. As the polarization ratio of fluorescence is an important quantity we have made a careful investigation of the fluorescence excited from anthracene crystals and also anthracene crystals containing small amounts of tetracene at room temperature, $-68^{\circ} \mathrm{C}$ and $-196^{\circ} \mathrm{C}$. We find that for single anthracene crystals (sublimation flakes) the polarization ratio $(b / a)$ is higher than in absorption at room temperature and is about $5: 1$. In extending these measurements to $-196^{\circ} \mathrm{C}$ we find no change in this ratio, however there is a shift of the spectrum to long wavelengths. In order to determine whether this shift was induced by changes in the dimensions of the unit cell at low temperatures, measurements were made on single crystals containing traces of tetracene. The tetracene fluorescence spectrum was however unchanged in position between room and low temperatures, so the shift of the anthracene fluorescence spectrum is to be correlated with the appearance of very weak absorption in the $4000 \mathrm{~A}$ region reported by previous workers. Although Sidman reports depolarization of the fluorescence of a number of solid solutions at low temperatures (mainly $20^{\circ} \mathrm{K}$ ) we failed to detect any such depolarization of the tetracene fluorescence which maintains its high room temperature value of between 4 and 5 to $1(b / a)$. If such a depolarization is present it must occur at lower temperatures than $77^{\circ} \mathrm{K}$, and our measurements are being extended to this region.

The high value of the polarization ratio compared to the absorption value could possibly be interpreted as evidence that the fluorescence arises mainly from those regions of the crystal where the lattice symmetry is disturbed, i.e, around dislocations. It is to be expected that for such regions crystal induced mixing of the ${ }^{1} B_{2 u}$ with the ${ }^{1} B_{31}$ state will be weakened with a resultant return to ${ }^{1} B_{2 u}$ character and the "oriented gas" value $(7: 1)$. This is consistent with the exciton treatment of the crystal levels.

In order to reconcile our results with those of Ganguly and Chaudhuri $(b / a, 1.54: 1)$, examination of the photograph reproduced in their paper shows that the crystals they used were so thick that the first two peaks of the fluorescence spectrum are not present and, evidently the peak that they observed (the third) also suffered from reabsorption $(b>a)$ which explains the low value they report.

All of the measurements were made using a Beckman DU spectrophotometer to disperse the fluorescence which was detected by a $1 \mathbf{P}-28$ photomultiplier. The fluorescence was excited by a BTU high pressure mercury lamp and a Wollaston prism was used to analyze the spectrum, only one beam being allowed to enter the spectrometer. For room temperature determinations the crystal was rotated through $90^{\circ}$ while for low temperatures the Wollaston was rotated through $180^{\circ} \mathrm{C}$.

I A. S. Davydov, J. Exptl. Theoret. Phys. (U.S.S.R.) 18, 210 (1948).

2 D. P. Craig and P. C. Hobbins, J. Chem. Soc. 1955, 2309 (1948).

3 D. P. Craig, J. Chem. Soc. 1955, 2302.

- A. V. Bree and L. E. Lyons, J. Chem. Soc. (to be published).

obreimov, Prikhotjko, and Shabaldas, J. Expt1. Theoret. Phys. (U.S.S.R.) 6, $1062(1936)$.

6 P. Pesteil and M. Barbaron, J. phys. radium 15, 92 (1954).

7 J. W. Sidman, Phys. Rev. 102, 96 (1956).

\& S. C. Ganguly and N. K. Chaudhuri, J. Chem. Phys. 19, 617 (1951).

\section{Raman Spectra of Phosphorous Trifluoride-Borane and Phosphorous Trifluoride-Borane- $\mathrm{d}_{3} \dagger$}

Robert C. TAYlor and Thomas C. Bissot

Department of Chemistry, University of Michigan, Ann Arbor, Michigan

(Received August 15, 1956)

$A^{\mathrm{L}}$

LTHOUGH borane, $\mathrm{BH}_{3}$, is not capable of existence in the free state, it is well known in compounds in which it acts as a Lewis acid. Recently, the preparation of the compound $\mathrm{PF}_{3} \mathrm{BH}_{3}$ has been reported ${ }^{1}$ and in view of the fact that in it $\mathrm{PF}_{3}$ exhibits an unexpected coordinating ability, it was felt there would be sufficient interest to justify a preliminary account of its Raman spectrum.

The reference to the preparation and properties of the normal and deuterated compounds has been cited; the samples after fractionation on the vacuum line were condensed into capillary Raman tubes which were then sealed off and maintained at liquid nitrogen temperatures until use. Details of the experimental 
TABLE I. Observed Raman frequencies and assignments for liquid $\mathrm{PF}_{3} \mathrm{BH}_{3}$ and $\mathrm{PF}_{3} \mathrm{BD}_{3}$ at $-80^{\circ} \mathrm{C}$.

\begin{tabular}{|c|c|c|c|c|c|}
\hline \multicolumn{2}{|c|}{$\begin{array}{l}\text { Frequency } \\
\text { in } \mathrm{cm}^{-1}\end{array}$} & \multirow{2}{*}{$\begin{array}{l}\text { Intensity } \\
\text { and } \\
\text { polariza- } \\
\text { tion }\end{array}$} & \multirow{2}{*}{\multicolumn{2}{|c|}{ Symmetry }} & \multirow[b]{2}{*}{ Assignment } \\
\hline $\mathrm{PF}_{3} \mathrm{BH}_{3}$ & $\mathrm{PF}_{3} \mathrm{BD}_{3}$ & & & & \\
\hline 197 & 169 & $m, d p$ & $e$ & $\nu_{12}$ & $\mathrm{PF}_{3}$ rock \\
\hline 370 & 362 & vw & $e$ & mi1 & $\mathrm{F}-\mathrm{P}-\mathrm{F}$ deformation \\
\hline 441 & 21 & $\mathrm{~m}, \mathrm{p}$ & $a_{1}$ & is & $F-P-F$ deformation \\
\hline 607 & 572 & $s, p$ & ai & 34 & $\mathrm{P} \rightarrow \mathrm{B}$ stretch \\
\hline $\begin{array}{l}697 \pm 2 \\
799\end{array}$ & $\cdots$ & vw & $e$ & vid & $\begin{array}{l}\mathrm{BH}_{8} \text { rock } \\
\text { diborane? }\end{array}$ \\
\hline $886 \pm 5$ & $\cdots$ & $v w, p ?$ & $A_{\mathrm{i}}$ & $2 \nu_{6}$ & $2 \times 441=882$ \\
\hline 920 & $\cdots$ & $\mathrm{w}, \mathrm{p}$ & $A_{1}+A_{2}+E$ & $\nu_{8}-\nu_{12}$ & $1117-197=920$ \\
\hline 944 & $944 \pm 5$ & $m, p ?$ & $a_{1}$ & $\nu_{s}$ & $\mathrm{P}-\mathrm{F}$ stretch \\
\hline $957 \pm 3$ & $958 \pm 2$ & $\mathrm{~m}, \mathrm{dp}$ & $e$ & 29 & $P-F$ stretch \\
\hline $1040=3$ & $\cdots$ & $w, p$ & At & $p_{4}+y_{6}$ & $441+607=1048$ \\
\hline 1077 & $\cdots$ & $w, p$ & $a_{1}$ & $\mu_{0}$ & $\mathrm{H}-\mathrm{B}-\mathrm{H}$ deformation \\
\hline 1117 & $\begin{array}{r}807 \\
1756\end{array}$ & $\begin{array}{l}\mathrm{s}, \mathrm{dp} \\
\mathrm{w}\end{array}$ & $A_{1}+A_{2}+E$ & ${ }_{\nu 8}^{\nu 8}$ & $\begin{array}{l}\mathrm{H}-\mathrm{B}-\mathrm{H} \text { deformation } \\
807+958=1765\end{array}$ \\
\hline$\cdots$ & 1797 & vow & & $\nu_{2}+\nu_{9} ?$ & $842+958=1800$ \\
\hline$\because$ & 1980 & vvw & & & diborane- $\mathrm{d}_{6}$ ? \\
\hline 2112 & $\cdots$ & vpw & & & diborane? \\
\hline $2140 \pm 4$ & $1672 \pm 2$ & vw & $A_{1}$ & $2 p z$ & $2 \times 1077=2154$ \\
\hline $2247 \pm 2$ & 1602 & vw & Al & $2 \psi_{\Omega}$ & $2 \times 1117=2234$ \\
\hline $2328 \pm 4$ & $\cdots$ & vow & & $2 w_{7}+\nu_{5} ?$ & cale 2329 \\
\hline 2385 & 1717 & vs, $p$ & $a_{1}$ & $\nu_{1}$ & $\mathrm{~B}-\mathrm{H}$ stretch \\
\hline 2455 & 1845 & $v s, d p$ & e & $\mu_{7}$ & $\overline{\mathrm{B}}-\mathrm{H}$ stretch \\
\hline$\cdots$ & 2431 & & & & B - H stretch \\
\hline 2530 & $\cdots$ & vvw & & & diborane? \\
\hline $2655 \pm 5$ & $\cdots$ & Vพw & $A_{1}+A_{2}+E$ & $p_{7}+v_{i}$ & $197+2455=2652$ \\
\hline
\end{tabular}

equipment have been given previously. ${ }^{2}$ The spectra were obtained of the liquid at $-80^{\circ} \mathrm{C}$, qualitative polarization measurements being made on the hydrogen compound only, using the two exposure method and polaroid cylinders. The data reported in Table I represent the averages from several spectra, the estimated probable error being approximately $1 \mathrm{~cm}^{-1}$ except where indicated.

Reference has been made previously ${ }^{1}$ to the chemical similarity between $\mathrm{PF}_{3}$ and carbon monoxide, and to the similarity in properties between phosphorous trifluoride-borane and carbon monoxide-borane. In view of the known structure of $\mathrm{BH}_{3} \mathrm{CO}_{3}{ }^{3}$ it would therefore be expected that the phosphorous complex would have an ethane type configuration with $C_{32}$ symmetry. The vibrational frequencies of such a structure are twelve in number and can be thought of in terms of the four vibrational frequencies of each of the two halves, considered as free molecules with $C_{30}$ symmetry, plus four vibrations arising as a consequence of the bond between the apex atoms of the two pyramids. Since free $\mathrm{BH}_{3}$ is not known, reference can be made to the Raman frequencies of $\mathrm{BH}_{3} \mathrm{CO}$ which have been determined recently. In the $\mathrm{B}-\mathrm{H}$ stretching region, two strong bands appear in the spectrum of $\mathrm{PF}_{3} \mathrm{BH}_{3}$ at 2385 and $2455 \mathrm{~cm}^{-1}$ which appear to be $a_{1}$ and $e$ type modes, respectively. Corresponding frequencies are found at 2380 and $2434 \mathrm{~cm}^{-1}$ in the spectrum of liquid $\mathrm{BH}_{3} \mathrm{CO}$. Boron-hydrogen deformation bands are observed at 1077 and $1117 \mathrm{~cm}^{-1}$ for the $a_{1}$ and $e$ modes which again do not differ greatly from the corresponding bands at 1073 and $1101 \mathrm{~cm}^{-1}$ in the carbon monoxide complex.

The frequencies associated with the $\mathrm{PF}_{3}$ group likewise show a close similarity in pattern to those found in the Raman spectrum of liquid $\mathrm{PF}_{3}$, which was also obtained in the present work. In this case, the $a_{1}$ and $e \mathrm{P}-\mathrm{F}$ stretching frequencies at 832 and $874 \mathrm{~cm}^{-1}$ shift to 994 and $958 \mathrm{~cm}^{-1}$, respectively, in the complex while the $a_{1}$ and $e$ bending frequencies shift from 484 and 351 to 441 and 370 $\mathrm{cm}^{-1}$, respectively.

The remaining modes may be described as a $\mathrm{P}-\mathrm{B}$ stretch, $\mathrm{BH}_{3}$ and $\mathrm{PF}_{3}$ rocking motions, and the inactive torsional mode. The first was easily identified as the strong polarized band at $607 \mathrm{~cm}^{-1}$, while the low depolarized band at $197 \mathrm{~cm}^{-1}$ is certainly the $\mathrm{PF}_{3}$ rock. The $\mathrm{BH}_{3}$ rock was assigned to the rather weak band at 697 $\mathrm{cm}^{-1}$. No information as to the torsional frequency was obtained.

Two fundamentals of $\mathrm{PF}_{3} \mathrm{BD}_{3}$ were not observed directly. The position of the first, the symmetrical $B-D$ bend, was estimated at $842 \mathrm{~cm}^{-1}$ from its first overtone, and the position of the second, the $\mathrm{BD}_{3}$ rock, was estimated at $603 \mathrm{~cm}^{-1}$ from the comparison with
$\mathrm{BD}_{3} \mathrm{CO} .{ }^{4}$ The calculated product rule ratios using these estimated values are 1.98 and 2.53 for the $a_{1}$ and $e$ classes which may be compared with the theoretical values of 1.97 and 2.55. The spectra of both molecules thus are interpreted satisfactorily in terms of the $C_{3 v}$ structure.

A normal coordinate treatment is in progress and its results together with a more detailed discussion of the assignments will be published on its completion.

* This research was conducted under Contract AF 23(616)-8 with the U. S. Air Force, the sponsoring agency being the Aeronautical Research Laboratory of the Wright Air Development Center, Air Research and Development Command.

i G. L. Vidale and R. C. Taylor, J. Am. Chem. Soc. 78, 294 (1956).

Z. W. Parry and T. C. Bissot, J. Am. Chem. Soc. 78, 1524 (1956).

${ }^{3}$ Gordy, Ring, and Burg, Phys. Rev, 78, 512 (1950),

4 R. C. Taylor (to be published).

\section{Butadiene Rydberg Spectrum}

\author{
ANDREW D. LIEHR* \\ Mallinckrodi Laboratory, Harward University. \\ Cambridge, Massachusetls \\ (Received July 30, 1956)
}

$\mathrm{T}$

HE far ultraviolet spectrum of butadiene has been investigated by W. C. Price and A. D. Walsh. ${ }^{1}$ It was found to consist of two Rydberg Patterns converging to a common limit. In this letter we wish to present a theoretical discussion of the butadiene Rydberg spectrum.

In the approximation of the semiempirical molecular orbital theory, butadiene is characterized by the ground states (1) cis ( $\left(A_{1}\right)$ $-\left(\psi b_{2}\right)^{2}\left(\psi a_{2}\right)^{2}$ and $(2)$ trans $\left({ }^{1} A_{0}\right)-\left(\psi a_{0}\right)^{2}\left(\psi b_{0}\right)^{2}$. These give rise to the Rydberg transitions (1) cis $-\psi a_{2} \rightarrow n p$, nd, etc., and (2) trans $-\psi b_{a} \rightarrow n p, n f$, etc. Now if for simplicity in calculating the term values we assume that butadiene has a linear carbon skeleton, we find that the hydrogenic energy levels split as follows: $n p$ becomes a doublet $\left(n p_{x}, n p_{y, x}\right), n d$ a triplet, $n f$ a quartet, and so forth. The additional splitting of the hydrogenic energy levels produced by the nonlinearity of the butadiene carbon skeleton is small.2 Thus, since only two pronounced Rydberg patterns have been found, ${ }^{3}$ we may identify the butadiene Rydberg transition as $\psi b_{\theta}($ trans $) \rightarrow n p($ trans $)+\psi a_{2}($ cis $) \rightarrow n p($ cis $)$. This assignment predicts a multiplet-like structure for the butadiene Rydberg series. ${ }^{3}$

The theoretical considerations given above for butadiene hold equally well for isoprene and the alkyl substituted butadienes. Hence, we would expect their spectra to be similar to that of butadiene. This similarity has been observed. ${ }^{1}$

* National Science Foundation Postdoctoral Fellow, 1955-1956. Now at Bell Telephone Laboratories, Murray Hill, New Jersey.

I W. C. Price and A. D. Walsh, Proc. Roy. Soc (London) A174, 220 (1940).

2 The calculation of $R$ ydberg term values for polyatomic molecules is described in A. D. Liehr, Z. Naturforsch (to be published).

* The small splitting of the nearly degenerate $p_{0}$ and $n p_{z}$ Rydberg levels and the excitation of torsional vibrations in the $n p$ Rydberg state should and the excitation of torsional vibrations in the $n p$ Rydberg state should This complexity has been noted by Price and Walsh. ${ }^{1}$

\section{On the Nuclear Physical Stability of the Uranium Minerals}

\section{P. K. KURODA}

Department of Chemistry, University of Arkansas, Fayetietille, Arkansas (Received July 26, 1956)

A $\mathrm{N}$ attempt is made in this paper to apply the nuclear reactor $A$ theory in geochronology and to explain certain interrelations between the age and the nuclear physical stability of the uranium minerals, as well as the geological environments of the mineral formation.

The infinite multiplication constant, $k_{x}$, may be considered as an indicator of the stability of the uranium minerals, which are the natural assemblages of uranium, moderator, and impurities. We 